\title{
SELF-SIMILAR SOLUTIONS IN REACTION-DIFFUSION SYSTEMS
}

\author{
JOANNA RENCŁAWOWICZ \\ Institute of Mathematics, Polish Academy of Sciences \\ Śniadeckich 8, P.O.Box 21, 00-956 Warszawa, Poland \\ E-mail:jr@impan.gov.pl
}

Abstract. In this paper we examine self-similar solutions to the system

$$
\begin{array}{ll}
u_{i t}-d_{i} \Delta u_{i}=\prod_{k=1}^{m} u_{k}^{p_{k}^{i}}, & i=1, \ldots, m, \quad x \in \mathbb{R}^{N}, \quad t>0, \\
u_{i}(0, x)=u_{0 i}(x), & i=1, \ldots, m, \quad x \in \mathbb{R}^{N},
\end{array}
$$

where $m>1$ and $p_{k}^{i}>0$, to describe asymptotics near the blow up point.

1. Introduction. In this paper we present the results that describe solutions to the system

$$
\begin{array}{ll}
u_{i t}-d_{i} \Delta u_{i}=\prod_{k=1}^{m} u_{k}^{p_{k}^{i}}, & i=1, \ldots, m, \quad x \in \mathbb{R}^{N}, \quad t>0, \\
u_{i}(0, x)=u_{0 i}(x), & i=1, \ldots, m, \quad x \in \mathbb{R}^{N},
\end{array}
$$

near the blow up point in terms of self-similar solutions to the system. Namely, we are going to show that blowing up solutions to the system are asymptotically close to the respective self-similar solutions. To formulate our results we have to introduce some convenient notation according to the system under consideration, i.e. (1.1).

We observe that without loss of generality we can assume henceforth that

$$
\min _{i} \sum_{k=1}^{m} p_{k}^{i}=\sum_{k=1}^{m} p_{k}^{1} .
$$

Let

$$
A_{m}=\left[p_{k}^{i}\right], \quad i, k=1, \ldots, m
$$

with row $i$ and column $k$.

2000 Mathematics Subject Classification: Primary 35B40; Secondary 35K55.

The paper is in final form and no version of it will be published elsewhere. 
Let

$$
\delta=\operatorname{det}\left(A_{m}-I\right)
$$

and suppose $\alpha=\left(\alpha_{1}, \ldots, \alpha_{m}\right)$ satisfies

$$
\left(A_{m}-I\right) \alpha^{t}=(1,1, \ldots, 1)^{t} .
$$

We notice that if $\delta \neq 0$ then the unique solution $\alpha$ of (1.5) is given by

$$
\alpha_{k}=\delta^{-1} \operatorname{det}\left(D_{k}\left(A_{m}-I\right)\right), \quad k=1, \ldots, m,
$$

where $D_{k}\left(A_{m}-I\right)$ denotes the matrix $A_{m}-I$ with column $k$ replaced by the vector $(1,1, \ldots, 1)^{t}$.

TheOREM 1.1 ([R3]). Let $u$ be a solution of $(1.1)$ defined in $\mathbb{R}^{N} \times(0, T)$. Assume that $\min _{i} \alpha_{i}>0$ and $\max _{i} \alpha_{i}=\alpha_{1}$, where the $\alpha_{i}$ are given by (1.3)-(1.6). Suppose that one of the conditions below holds:

$$
\begin{gathered}
N=1,2 \quad \text { or } \quad N \geq 3, \alpha_{1} \geq \frac{N-2}{4}, \\
d_{1} \Delta u_{0_{1}}+A_{1} u_{0_{1}}^{1+1 / \alpha_{1}}>0 .
\end{gathered}
$$

Then for some constant $C>0$,

$$
u_{i}(x, t) \leq C(T-t)^{-\alpha_{i}}, \quad i=1, \ldots, m .
$$

This result makes it possible to proceed further in order to derive a finer description of a solution near a blow up. Our approach starts with introducing similarity variables in the following way:

$$
\begin{aligned}
& u_{i}(x, t)=(T-t)^{-\alpha_{i}} U_{i}(y, s), \\
& y=x(T-t)^{-1 / 2}, \quad s=-\log (T-t) .
\end{aligned}
$$

Then the new functions $U_{i}$ solve

$$
U_{i s}=d_{i} \Delta U_{i}-\frac{1}{2} y \nabla U_{i}-\alpha_{i} U_{i}+\prod_{k=1}^{m} U_{k}^{p_{k}^{i}}, \quad i=1, \ldots, m .
$$

Our task is to characterize all global bounded nonnegative solutions of the rescaled system (1.11). Since $t \rightarrow T$ corresponds to $s \rightarrow \infty$, the analysis of nonglobal solutions of (1.1) is now equivalent to examining the large-time asymptotics of solutions of (1.11). However, the last problem seems to be easier to solve. Consequently, our task is to characterize all global bounded nonnegative solutions of the rescaled system (1.11). It turns out that under some restriction on $p_{k}^{i}$ such solutions are constant. We can divide the argument into two steps. We start with the assuring ourselves that as $s \rightarrow \infty, U_{i}(y, s)$ are independent of $s$, i.e. should approach a stationary solution of the system. Next, we characterize such solutions as the constants. In this way we will show that the solution $u$ of (1.1) is "asymptotically self-similar". Our main result is

THEOREM 1.2. Let $U=\left(U_{1}, \ldots, U_{m}\right)$ be any nonnegative bounded solution of $(1.11)$ defined for $(y, s) \in \mathbb{R}^{N} \times \mathbb{R}$. Assume that $U \not \equiv \mathbf{0}$ where $\mathbf{0}=(0, \ldots, 0)$ and $\max _{i} \alpha_{i}=\alpha_{1}$. If

$$
\min _{i} \alpha_{i}>0
$$


and

$$
N=1,2
$$

or

$$
N \geq 3,1<\frac{\sum_{k=i}^{m} p_{k}^{i} \alpha_{k}}{\alpha_{i}} \leq \frac{N+2}{N-2}, \quad i=1, \ldots, m,
$$

then

$$
\lim _{s \rightarrow \infty}\left(U_{1}, \ldots, U_{m}\right)=\left(C_{\alpha_{1}}, \ldots, C_{\alpha_{m}}\right)
$$

where $\left(C_{\alpha_{1}}, \ldots, C_{\alpha_{m}}\right)$ are constant solutions of the system (1.11).

We emphasize that the assumptions of Theorem 1.2 imply the growth rate estimates (1.9) since condition (1.7) is satisfied. Therefore $U_{i}$ can be defined by the system (1.1) and formulas (1.10).

2. Preliminaries. First, we recall some auxiliary facts established in [R3] and [R2]. We put

$$
b_{k}=\frac{\alpha_{k}}{\alpha_{1}}, \quad k=1, \ldots, m,
$$

assuming that $\alpha_{1} \neq 0$ and $b_{k}>0$ (the last condition is guaranteed if $\min _{k} \alpha_{k}>0$ ). We also define

$$
r=\sum_{k=1}^{m} b_{k} p_{k}^{1}=\frac{1+\alpha_{1}}{\alpha_{1}} .
$$

Let us consider the following kinetic system corresponding to (1.1):

$$
\left\{\begin{array}{l}
u_{i}^{\prime}=\prod_{k=1}^{m} u_{k}^{p_{k}^{i}} \\
u_{i}(0)=u_{0 i},
\end{array} \quad i=1, \ldots, m .\right.
$$

Lemma 2.1. The set

$$
\begin{aligned}
\partial M=\left\{\left(u_{1}, \ldots, u_{m}\right):\right. & u_{i} \geq 0, F\left(u_{1}, \ldots, u_{m}\right)=\left(F_{1} \ldots, F_{m-1}\right)\left(u_{1}, \ldots, u_{m}\right)=\mathbf{0} ; \\
& \left.F_{j}\left(u_{1}, \ldots, u_{m}\right)=u_{j+1}-a_{j+1} u_{j}^{b_{j+1} / b_{j}}, \quad j=1, \ldots, m-1\right\},
\end{aligned}
$$

where $a_{j}$ are constants given by the conditions

$$
a_{1}=1, \quad b_{i} \prod_{j=1}^{i} a_{j}^{b_{i} / b_{j}}=\prod_{k=1}^{m}\left(\prod_{j=1}^{k} a_{j}^{b_{k} / b_{j}}\right)^{p_{k}^{i}}, \quad i=2, \ldots, m .
$$

is an invariant manifold for (2.3).

Definition 2.1 (Invariant region). Let $\frac{d u}{d t}+A u=f(t, u), u=\left(u_{1}, \ldots, u_{m}\right)$. A region $D$ is called a regular invariant region for this system, if the conditions $u_{0} \in C^{2}$, $u_{0} \in \partial D$ imply that $(t, u(x, t)) \in D$ (where $u$ denotes the solution of this system). 
LEMMA 2.2. Let

$$
\begin{aligned}
& u=\left(u_{1}, \ldots, u_{m}\right), \quad F(u)=\left(F_{1}(u), \ldots, F_{m-1}(u)\right) \\
& \quad F_{i}(u)=u_{i+1}-a_{i+1} u_{i}^{b_{i+1} / b_{i}}, \quad i=1, \ldots, m-1
\end{aligned}
$$

and

$$
\begin{aligned}
& M=\left\{u: F(u) \leq \mathbf{0}, u_{i} \geq 0, i=1, \ldots, m\right. \\
&\text { and } \left.\left(d_{i+1}-d_{i}\right) \Delta u_{0 i} \leq 0 \text { for } u_{0 i}=u_{i}(0) \in C^{2}\right\} .
\end{aligned}
$$

Then $M$ is a regular invariant region for (1.1) if $\frac{b_{i+1}}{b_{i}} \leq 1$ for all $i=1, \ldots, m-1$ (i.e., $u_{0} \in C^{2}, u_{0} \in \partial M$ imply that $u(x, t) \in M$ for all $\left.t<T\right)$.

3. Asymptotical self-similarity of solutions. In this section we consider the natural question about a finer description of $u$ near blow up. Assuming estimates on the growth rate established in [R3], i.e.

$$
u_{i}(x, t) \leq C(T-t)^{-\alpha_{i}}, \quad i=1, \ldots, m .
$$

we define the following change of both independent and dependent variables

$$
\begin{aligned}
& U_{i}(y, s)=(T-t)^{\alpha_{i}} u_{i}(x, t) \\
& y=x(T-t)^{-1 / 2}, \quad s=-\log (T-t) .
\end{aligned}
$$

We introduce the system corresponding to new functions and variables called the similarity variables

$$
U_{i s}=d_{i} \Delta U_{i}-\frac{1}{2} y \nabla U_{i}-\alpha_{i} U_{i}+\prod_{k=1}^{m} U_{k}^{p_{k}^{i}}, \quad i=1, \ldots, m .
$$

We argue by means of the invariant region technique. The utility of the method consists in the possibility of rewriting the system (1.1) to a system of inequalities. Nevertheless, all are the scalar ones. Moreover, it turns out that adapting a technique used to discuss the scalar problem is then available.

Applying Lemma 2.2 we can rewrite the system (1.1) (so respectively (3.2)) as follows, provided that initial values belong to $\partial M$ :

$$
u_{i t}-d_{i} u_{i} \leq M_{i} u_{1}^{\sum_{k=1}^{i-1} b_{k} p_{k}^{i}} u_{i}^{\sum_{k=i}^{m} b_{k} p_{k}^{i} / b_{i}}, \quad i=1, \ldots, m
$$

and

$$
\begin{aligned}
U_{i s}-d_{i} \Delta U_{i}+\frac{1}{2} y \nabla U_{i}+\alpha_{i} U_{i} & \leq M_{i} \prod_{k=1}^{i-1} U_{1}^{b_{k} p_{k}^{i}} \prod_{k=i}^{m} U_{i}^{b_{k} p_{k}^{i} / b_{i}} \\
& =M_{i} U_{1}^{\sum_{k=1}^{i-1} b_{k} p_{k}^{i}} U_{i}^{\sum_{k=i}^{m} b_{k} p_{k}^{i} / b_{i}}
\end{aligned}
$$


where

$$
\begin{gathered}
M_{1}=A_{1}=\prod_{k=1}^{m}\left(\prod_{j=1}^{k} a_{j}^{b_{k} / b_{j}}\right)^{p_{k}^{i}} \\
M_{i}=\prod_{k=1}^{i-1}\left(\prod_{j=1}^{k} a_{j}^{b_{k} / b_{j}}\right)^{p_{k}^{i}} \prod_{k=i}^{m}\left(\prod_{j=i}^{k} a_{j}^{b_{k} / b_{j}}\right)^{p_{k}^{i}}, \quad i=2, \ldots, m .
\end{gathered}
$$

We now focus on the first inequality of (3.4) which in fact is scalar.

$$
U_{1 s}-d_{1} \Delta U_{1}+\frac{1}{2} y \nabla U_{1}+\alpha_{1} U_{1}-M_{1} U_{1}^{\left(1+\alpha_{1}\right) / \alpha_{1}} \leq 0 .
$$

From here we pursue the existing trail for the scalar equation. We refer also to Section 3 in [R4], where the case $m=2, d_{i}=1$ is discussed. According to this problem we start with the assuring ourselves that as $s \rightarrow \infty, U_{1}$ is necessarily self-similar, i.e. independent of $s$. Therefore, $U_{1}$ should approach a stationary solution of (3.6). From this point of view we shall next analyze the global nonnegative and bounded solutions of the equation

$$
d_{1} \Delta U_{1}-\frac{1}{2} y \nabla U_{1}-\alpha_{1} U_{1}+M_{1} U_{1}^{\left(1+\alpha_{1}\right) / \alpha_{1}}=0 .
$$

It turns out that they are constants. We set

$$
\begin{aligned}
& U_{1}^{+\infty}(y, s)=\lim _{s_{j} \rightarrow \infty} U_{1}\left(y, s+s_{j}\right) \\
& U_{1}^{-\infty}(y, s)=\lim _{s_{j}^{\prime} \rightarrow-\infty} U_{1}\left(y, s+s_{j}^{\prime}\right) .
\end{aligned}
$$

This corresponds to limits $\lim _{t \rightarrow T} u_{1}(x, t)$ and $\lim _{t \rightarrow-\infty} u_{1}(x, t)$, respectively.

The first step of our analysis can be expressed as follows:

Proposition 3.1. If $U_{1}$ is a bounded global solution of (3.6), then both limits $U_{1}^{\infty}$ and $U_{1}^{-\infty}$ exist and are independent of $s$. Moreover, $U_{1}\left(y, s+s_{j}\right) \rightarrow U_{1}^{ \pm \infty}$ as $s_{j} \rightarrow \pm \infty$ uniformly on compact subsets of $\mathbb{R}^{N}$ with $\lim _{s \rightarrow \pm \infty} \nabla U_{1}(y, s)=0$ for almost every $y$.

Proof. Since the approach is completely parallel with the case of the single equation, we shall just recall main ideas, pointing out differences emerging for the inequality (3.6).

We quote a technical result of Giga and Kohn ([GK1], Proposition 1).

LEMMA 3.2. If $u_{1}$ is a function satisfying (3.3) and if (1.9) holds, then

$$
\left|\nabla^{k} u_{1}(x, t)\right| \leq C(T-t)^{-\beta-k / 2} \text {. }
$$

This implies

LEMMA 3.3. If $U_{1}$ is a bounded function satisfying (3.6) defined on $\mathbb{R}^{N+1}$ then

$$
\left|\nabla U_{1}\right|+\left|\nabla^{2} U_{1}\right| \leq C^{\prime}, \quad\left|U_{1_{s}}\right| \leq C^{\prime}(1+|y|)
$$

Our problem can be represented as

$$
\rho U_{1 s}-d_{1} \nabla \cdot\left(\rho \nabla U_{1}\right)+\frac{1}{r-1} \rho U_{1}-M_{1} \rho U_{1}^{r} \leq 0,
$$

where $r=1+\frac{1}{\alpha_{1}}, \rho(y)=\exp \left(-\frac{1}{4}|y|^{2}\right)$. We claim 
LEMMA 3.4. If $U_{1}$ solves (3.6) and is bounded then

$$
\int_{a}^{b} \int_{\mathbb{R}^{N}}\left|U_{1 s}\right|^{2} \rho d y d s \leq E\left[U_{1}\right](a)-E\left[U_{1}\right](b),
$$

where $a<b$ are real and

$$
\begin{aligned}
& E\left[U_{1}\right](s)=\frac{d_{1}}{2} \int_{\mathbb{R}^{N}}\left|\nabla U_{1}\right|^{2} \rho d y \\
& \quad+\frac{1}{2(r-1)} \int_{\mathbb{R}^{N}}\left|U_{1}\right|^{2} \rho d y-\frac{M_{1}}{r+1} \int_{\mathbb{R}^{N}}\left|U_{1}\right|^{r+1} \rho d y .
\end{aligned}
$$

Proof. We derive the above assertion analogously to Proposition 3 in [GK1]. Multiplying (3.6) by $U_{1 s}$ and integrating over $B_{R}=\{y:|y|<R\}$ one gets

$$
\begin{aligned}
& \int_{B_{R}}\left|U_{1 s}\right|^{2} \rho d y \\
& \leq \int_{B_{R}}\left(d_{1} U_{1 s} \nabla \cdot\left(\rho \nabla U_{1}\right)-\frac{1}{2(r-1)} \frac{d}{d s}\left|U_{1}\right|^{2} \rho+\frac{M_{1}}{r+1} \frac{d}{d s}\left|U_{1}\right|^{r+1} \rho\right) d y \\
& =-\frac{d_{1}}{2} \frac{d}{d s} \int_{B_{R}}\left|\nabla U_{1}\right|^{2} \rho d y+\int_{\partial B_{R}} U_{1 s} \frac{\partial U_{1}}{\partial r} \rho d \sigma \\
& \quad-\frac{d_{1}}{2(r-1)} \frac{d}{d s} \int_{B_{R}}\left|U_{1}\right|^{2} \rho d y+\frac{M_{1}}{r+1} \frac{d}{d s} \int_{B_{R}}\left|U_{1}\right|^{r+1} \rho d y .
\end{aligned}
$$

By Lemma 3.3 we observe that the surface term tends to zero as $R \rightarrow \infty$. Therefore, the integration from $a$ to $b$ and the passage with $R$ to the limit gives

$$
\int_{a}^{b} \int_{\mathbb{R}^{N}}\left|U_{1 s}\right|^{2} \rho d y d s \leq E\left[U_{1}\right](a)-E\left[U_{1}\right](b) .
$$

This concludes the proof.

The last inequality is basic to the further elaboration.

LEMMA 3.5. Assume that $U_{1}$ is a bounded solution of (3.6) on $\mathbb{R}^{N+1}$ and a sequence $s_{j} \rightarrow+\infty$ (respectively, $s_{j} \rightarrow-\infty$ ) is monotonically increasing (decreasing) with $s_{j+1}-s_{j} \rightarrow \pm \infty$ as $j \rightarrow \infty$. Let $U_{1}^{j}(y, s)=U_{1}\left(y, s+s_{j}\right)$ converge to a limit $U_{1}^{ \pm \infty}$ uniformly on compact sets of $\mathbb{R}^{N+1}$ with $\nabla U_{1}^{j}(y, m) \rightarrow \nabla U_{1}^{ \pm \infty}(y, m)$ almost everywhere in $\mathbb{R}^{N}$ for all integer $m$. Then $U_{1}^{ \pm \infty}$ does not depend on $s$ and $E\left[U_{1}^{\infty}\right], E\left[U_{1}^{-\infty}\right]$ are independent of the choice of $\left\{s_{j}\right\}$.

Proof. This lemma is an analogue of Proposition 4 in [GK1]. We consider $s_{j} \rightarrow \infty$. By Lemma 3.4, putting $U_{1}=U_{1}^{j}, a=m, b=m+s_{j+1}-s_{j}$ in (3.11) we get

$$
\begin{array}{r}
\int_{m}^{m+s_{j+1}-s_{j}} \int_{\mathbb{R}^{N}}\left|U_{1 s}^{j}\right|^{2} \rho d y d s \leq E\left[U_{1}^{j}\right](m)-E\left[U_{1}^{j}\right]\left(m+s_{j+1}-s_{j}\right) \\
=E\left[U_{1}^{j}\right](m)-E\left[U_{1}^{j+1}\right](m) .
\end{array}
$$

Lemma 3.3 yields that $\left|\nabla U_{1}^{j}\right|<C,\left|U_{1}^{j}\right|<c$ for all $i$. Thus, we can pass with $j \rightarrow \infty$ in (3.15) and since $E\left[U_{1}^{j}\right](m) \rightarrow E\left[U_{1}^{\infty}\right](m), s_{j+1}-s_{j} \rightarrow \infty$, we see that

$$
\lim _{j \rightarrow \infty} \int_{m}^{K} \int_{\mathbb{R}^{N}}\left|U_{1 s}^{j}\right|^{2} \rho d y d s \leq 0, \quad m<K .
$$


Therefore, we must have

$$
\lim _{j \rightarrow \infty} \int_{m}^{K} \int_{\mathbb{R}^{N}}\left|U_{1 s}^{j}\right|^{2} \rho d y d s=0 .
$$

It follows by $\left|U_{1 s}^{j}\right| \leq C(1+|y|)$ (i.e. (3.9)) and a lower-semicontinuity of the integral in (3.17) that also

$$
\int_{m}^{K} \int_{\mathbb{R}^{N}}\left|U_{1 s}^{\infty}\right|^{2} \rho d y d s=0
$$

for even $m$ and $K$, so $U_{1}^{\infty}$ is necessarily independent of $s$.

Now, we discuss properties of $E\left[U_{1}^{\infty}\right]$. We argue by contradiction. Let $\breve{s}_{j}$ be such a sequence that $\breve{s}_{j}$ satisfies assumptions of Lemma 3.5, $\breve{U}_{1}^{j}(y, s)=U_{1}\left(y, s+\breve{s}_{j}\right), \breve{U}_{1}^{\infty}=$ $\lim _{\breve{s}_{j} \rightarrow \infty} \breve{U}_{1}^{j}$ and $E\left[U_{1}^{\infty}\right] \neq E\left[\breve{U}_{1}^{\infty}\right]$. Let $E\left[U_{1}^{\infty}\right] \leq E\left[\breve{U}_{1}^{\infty}\right], s_{j}<\breve{s}_{j}$ (we can obtain it passing to a subsequence). We use Lemma 3.4 setting in (3.11) $a=s_{j}, b=\breve{s}_{j}$. Then

$$
\begin{aligned}
\int_{s_{j}}^{\breve{s}_{j}} \int_{\mathbb{R}^{N}}\left|U_{1 s}\right|^{2} \rho d y d s \leq & E\left[U_{1}\right]\left(s_{j}\right)-E\left[U_{1}\right]\left(\breve{s}_{j}\right)= \\
& =E\left[U_{1}^{j}\right](0)-E\left[\breve{U}_{1}^{j}\right](0) \rightarrow E\left[U_{1}^{\infty}\right]-E\left[\breve{U}_{1}^{\infty}\right]<0 .
\end{aligned}
$$

Thus, for $j$ large enough, there is a contradiction with a nonnegativity of the integral in (3.19). We conclude that $E\left[U_{1}^{\infty}\right]=E\left[\breve{U}_{1}^{\infty}\right]$, i.e. $E\left[U_{1}^{\infty}\right]$ is independent of $\breve{s}_{j}$. We complete the proof considering the case $s_{j} \rightarrow-\infty$ similarly.

Finally, by (3.9) we have

$$
\left|\nabla U_{1}\right| \leq C^{\prime}, \quad\left|U_{1 s}\right| \leq C^{\prime}(1+|y|)
$$

then for some subsequence $\left\{s_{j}\right\}, s_{j} \rightarrow \infty, U_{1}\left(y, s+s_{j}\right) \rightarrow U_{1}^{\infty}(y, s)$ uniformly on compact sets. The bound $\left|\nabla^{2} U_{1}\right| \leq C$ assures that $\nabla U_{1}\left(y, m+s_{j}\right) \rightarrow \nabla U_{1}^{\infty}(y, m)$ almost everywhere for every integer $m$ and some subsequence $s_{j}$. Clearly, we can take such a subsequence to $s_{j+1}-s_{j} \rightarrow \infty$, also. Then we obtain a desired conclusion.

Summarizing Lemmas 3.4 and 3.5, we infer Proposition 3.1.

To complete the classification of bounded solutions of (3.2) we prove that such solutions of corresponding stationary problem are constant.

Proposition 3.6. If $U_{1}$ is a stationary, global and bounded solution of (3.6), so satisfies

$$
d_{1} \Delta U_{1}-\frac{1}{2} y \nabla U_{1}-\frac{U_{1}}{r-1}+M_{1} U_{1}^{r} \geq 0
$$

then $U_{1}$ is a constant such that $U_{1} \leq\left[M_{1}(r-1)\right]^{-1 /(r-1)}$ provided $N=1,2$ or $N \geq 3$ and $1<r<\frac{N+2}{N-2}$ (i.e. $\left.\alpha_{1} \geq \frac{N-2}{4}\right)$.

Proof. As we have mentioned, $U_{1}$ is a subsolution of a corresponding scalar equation, namely $U_{1}(y) \leq \bar{U}_{1}(y)$, where

$$
d_{1} \Delta \bar{U}_{1}-\frac{y}{2} \nabla \bar{U}_{1}-\frac{\bar{U}_{1}}{r-1}-M_{1} \bar{U}_{1}^{r}=0 .
$$


However, the above problem has been studied by several authors, so we can employ the known result (cf. Theorem 1 of [GK1], also [GS]) to infer that (3.21) has only constant global solutions: $\bar{U}_{1} \equiv 0$ or $\bar{U}_{1} \equiv K$, where

$$
K=\left(\frac{\alpha_{1}}{M_{1}}\right)^{\alpha_{1}}=\left[M_{1}(r-1)\right]^{-1 /(r-1)}
$$

provided $N=1,2$ or $N \geq 3,1<r<\frac{N+2}{N-2}$.

We slightly transform (3.20) into

$$
\nabla \cdot\left(\rho \nabla U_{1}\right)-\frac{\rho}{r-1} U_{1}+M_{1} \rho\left|U_{1}\right|^{r} \geq 0
$$

with $\rho(y)=\exp \left(-\frac{1}{4}|y|^{2}\right)$. Multiplying (3.23) by $-U_{1}$, integrating over $\mathbb{R}^{N}$ and integrating by parts in the first term, we get

$$
\int_{\mathbb{R}^{N}}\left|\nabla U_{1}\right|^{2} \rho d y+\frac{1}{r-1} \int_{\mathbb{R}^{N}}\left|U_{1}\right|^{2} \rho d y-M_{1} \int_{\mathbb{R}^{N}}\left|U_{1}\right|^{r+1} \rho d y \leq 0 .
$$

This yields

$$
\int_{\mathbb{R}^{N}}\left|\nabla U_{1}\right|^{2} \rho d y \leq \int_{\mathbb{R}^{N}}\left|U_{1}\right|^{2} \rho\left(M_{1}\left|U_{1}\right|^{r-1}-\frac{1}{r-1}\right) d y
$$

Consequently, by $\left|U_{1}\right| \leq\left[\frac{1}{M_{1}(r-1)}\right]^{1 /(r-1)}$ or $\left|U_{1}\right|=0$ we obtain

$$
\int_{\mathbb{R}^{N}}\left|\nabla U_{1}\right|^{2} \rho d y \leq 0 \quad \text { or } \quad U_{1} \equiv 0
$$

Thus $U_{1}$ is some constant. Moreover, $U_{1} \equiv 0$ or $0<U_{1} \leq K=\left[M_{1}(r-1)\right]^{-1 /(r-1)}$ if we assume that $r$ is subcritical.

Now we return to the system (3.4). If one considers the asymptotic behavior of $U_{i}$ as $s \rightarrow \infty$, then the assertion proved for $U_{1}$ yields

$$
U_{i s}-d_{i} \Delta U_{i}+\frac{y}{2} \nabla U_{i}+\alpha_{i} U_{i} \leq M_{i} K^{\sum_{k=1}^{i-1} b_{k} p_{k}^{i}} U_{i}^{\sum_{k=i}^{m} b_{k} p_{k}^{i} / b_{i}}, \quad i=2, \ldots, m
$$

where $K$ is given by (3.22).

Therefore, for the problem (3.27) for each $i$ we can repeat the analysis analogous to that shown for (3.6) in the above Propositions and Lemmas, to conclude that $U_{i}(y, s)$ $(i=2, \ldots, m)$ should approach, as $s \rightarrow \infty$, a stationary solution of (3.27) and then, if $1<\sum_{k=i}^{m} \frac{b_{k}}{b_{i}} p_{k}^{i} \leq \frac{N+2}{N-2}, N \geq 3$ or $N=1,2$, the only global bounded nonnegative solution of (3.27) independent of $s$ is a constant. Moreover, since $y$ is the blow up point, the argument parallel to that presented for the equation in [GK3] excludes the trivial solution. On the other hand, we know that $U_{i}$ are the solutions of system (3.2). Then $U_{i} \rightarrow C_{\alpha_{i}}$ as $s \rightarrow \infty$, where $C_{\alpha_{i}}$ are constants satisfying (3.2).

It remains to give some thought to the case when initial data do not belong to $\partial M$. Then one can find $\bar{u}_{0_{1}}$ and $\underline{u}_{0_{1}}$ such that

$$
u_{0 i} \leq \bar{u}_{0 i}=\prod_{j=1}^{i} a_{j}^{b_{i} / b_{j}}\left(\bar{u}_{0_{1}}\right)^{b_{i}}
$$


and

$$
\underline{u}_{0 i}=\prod_{j=1}^{i} a_{j}^{b_{i} / b_{j}}\left(\underline{u}_{0_{1}}\right)^{b_{i}} \leq u_{0 i} .
$$

Clearly, as $\bar{u}_{i}$ and $\underline{u}_{i} \in \partial M$, we conclude the same asymptotics properties as above for corresponding $\bar{U}_{i}$ and $\underline{U}_{i}$. Moreover by comparison

$$
\underline{U}_{\infty} \leq U_{\infty} \leq \bar{U}_{\infty}
$$

In this way we have showed that the solution $u$ of (1.1) is "asymptotically self-similar". Thus, the proof is complete.

\section{References}

[AHV] D. Andreucci, M. A. Herrero and J. J. L. Velázquez, Liouville theorems and blow up behaviour in semilinear reaction diffusion systems, Ann. Inst. H. Poincaré Anal. Non Linéaire 14 (1997), 1-53.

[AD] D. Andreucci and E. DiBenedetto, On the Cauchy problem and initial traces for a class of evolution equations with strongly nonlinear sources, Ann. Scuola Norm. Sup. Pisa Cl. Sci. (4) 18 (1991), 363-441.

[CM] G. CARISTi and E. Mitidieri, Blow-up estimates of positive solutions of a parabolic system, J. Differential Equations 113 (1994), 265-271.

[EL] M. Escobedo and H. A. Levine, Critical blowup and global existence numbers for a weakly coupled system of reaction-diffusion equations, Arch. Rational Mech. Anal. 129 (1995), 47-100.

[FM] A. Friedman and B. MCLEOD, Blow-up of positive solutions of semilinear heat equations, Indiana Univ. Math. J. 34 (1985), 425-447.

[GS] B. GIDAS and J. SpRUCK, Global and local behaviour of positive solutions of nonlinear elliptic equations, Comm. Pure Appl. Math. 34 (1981), 525-598.

[GK1] Y. Giga and R. V. Konn, Asymptotically self-similar blow-up of semilinear heat equations, Comm. Pure Appl. Math. 38 (1985), 297-319.

[GK2] Y. GigA and R. V. KonN, Characterizing blowup using similarity variables, Indiana Univ. Math. J. 36 (1987), 1-40.

[GK3] Y. Giga and R. V. KoHn, Nondegeneracy of blowup for semilinear heat equations, Comm. Pure Appl. Math. 42 (1989), 845-884.

[HV1] M. A. Herrero and J. J. L. Velázquez, Blow-up behaviour of one-dimensional semilinear parabolic equations, Ann. Inst. H. Poincaré Anal. Non Linéaire 10 (1993), 131-189.

[HV2] M. A. Herrero and J. J. L. Velázquez, Flat blow-up in one-dimensional semilinear heat equations, Differential Integral Equations 5 (1992), 973-997.

[L] G. Lu, Global existence and blow-up for a class of semilinear parabolic systems: a Cauchy problem, Nonlinear Anal. 24 (1995), 1193-1206.

[R1] J. RencŁaWowicz, Global existence and blow up of solutions for a completely coupled Fujita type system of reaction-diffusion equations, Appl. Math. (Warsaw) 25 (1998), 313-326.

[R2] J. RencŁawowicz, Global existence and blow up for a completely coupled Fujita type system, Appl. Math. (Warsaw) 27 (2000), 203-218. 
[R3] J. RencŁawowicz, Blow up, global existence and growth rate estimates in nonlinear parabolic systems, Colloq. Math. 86 (2000), 43-66.

[R4] J. RENCŁAWOWICZ, Asymptotic behaviour of solutions of parabolic reaction-diffusion systems, Differential Integral Equations 15 (2002), 191-212.

[V] J. J. L. VelázQuez, Classification of singularities for blowing up solutions in higher dimensions, Trans. Amer. Math. Soc. 338 (1993), 441-464. 